\title{
External and internal fixation for comminuted intra-articular fractures of distal radius
}

\author{
Pradhan RL, Lakhey S, Pandey BK, Manandhar RR, Rijal KP, Sharma S \\ Department of Orthopaedics, Kathmandu Medical College, Sinamangal, Nepal
}

\begin{abstract}
Background: Distal radius fractures are a common injury and without proper treatment leads to high functional impairment and frequent complications.

Objective: The aim of this study was to see the functional outcome in patients with comminuted distal radius fractures treated with combined external fixation and open reduction with volar plating.

Materials and methods: All comminuted distal radius fractures classified as type $\mathrm{C}$ in AO/OTA classification were enrolled for the study from 2005 till 2008. The clinical scoring chart modified by Cooney was used to evaluate the functional outcome.

Results: There were twenty-two patients with the average age of 42.18 years (range 19-60) with 15 male and 7 females. The follow-up period was from 14 to 46 months. Accordingly, there were 11 (50\%) excellent, 7 (31\%) good, 2 (9\%) fair and $2(9 \%)$ poor results. There were very few complications in our series. In three patients additional K-wire supplementation was necessary along with external and open internal fixation.

Conclusions: Comminuted intra-articular fractures of distal radius should be treated by open reduction and combined internal and external fixation to achieve a high rate of patient satisfaction and satisfactory functional outcome.
\end{abstract}

Key words: distal radius fractures, volar plating, external fixation

$\mathrm{U}$ nstable or fractures that involve the articular surfaces of the distal radius can jeopardise the congruence and kinematics of the wrist joint. Anatomical articular reduction greatly reduces the incidence of post-traumatic osteoarthrosis and that the quality of reduction relates directly to the final outcome ${ }^{1}$. These types of fractures have been treated by various methods of treatment methods. Many fixation techniques have been described including pin and plaster fixation, percutaneous pinning, intramedullary pinning, external fixation (bridging or nonbridging, static or dynamic) and various forms of internal fixation with customised implants. The advantages of external fixation are the relative ease of application, minimal surgical exposure, and reduced surgical trauma. The disadvantages are prolonged immobilisation of the radiocarpal joint, indirect reduction of fracture fragments, loss of ligamentotaxis over time, and pinrelated complications ${ }^{2,3,4}$. The advantages of plate osteosynthesis are direct fracture reduction, stable rigid fixation, and the possibility of immediate postoperative motion $^{5,6,7}$. An external fixator can be applied to any fracture pattern but it functions poorly for shearing fractures in the coronal plane. External fixation without any interfragmentary pins provides at best an indirect reduction without a means to maintain that reduction.
Traction may not however reduce separated and rotated intra-articular radius fractures, and open reduction with internal fixation is required. Open reduction of the fracture allows reduction of the fracture under direct vision and should lead to accurate restoration of anatomy. Dorsal plates were associated with tendon rupture, tenosynovits, reoperation, and $25 \%$ of collapse while the complication of volar plating is relatively is low ${ }^{7}$. The volar surface of the distal radius may be biologically more acceptable for open reduction and internal fixation than the dorsal surface because the flexor tendons are not as close to the bone surface as the extensor tendons. Buttress plates reduce and stabilise vertical shear intra-articular fractures through an antiglide effect whereas conventional plates address metaphyseal comminution and preserve articular congruity and reduction ${ }^{8}$.

Combined internal and external fixation of distal radius fractures is used most commonly to treat injuries with

\section{Correspondence}

Rabindra L Pradhan

Department of Orthopaedic Surgery

Kathmandu Medical Teaching Hospital

Sinamangal, Kathmandu, Nepal

E-mail: rabi.ortho@gmail.com 
joint surface or metaphyseal comminution. External fixation aids reduction intraoperatively and facilitates percutaneous, or open manipulation of the fracture. Internal fixation maintains precise reduction of critical anatomy, principally the contour and orientation of the articular surface. Postoperatively the fixator functions as a neutralisation device, preventing fracture collapse and decreasing the biomechanical demands on the internal fixation hardware. The combined technique exploits the benefits of both forms of fixation, allowing each to be used to full advantage in the treatment of complex distal radius fractures ${ }^{9}$.

Combinations of internal and external fixation to more accurately reduce the more severely fractured distal radius have been described in the literature. However these studies have used various methods of internal fixation ranging from $\mathrm{K}$ wire, to volar and dorsal plating. Our study reports on a surgical approach for the management of comminuted distal radius fractures that combines open reduction and internal fixation with volar plates and the use of external fixation.

\section{Materials and methods}

During the study period from 2005 till 2008 all patients with comminuted distal radius coming to Kathmandu Medical College Teaching Hospital were evaluated and those that needed treatment with external and open reduction and internal fixation formed the basis of this study. Patients treated with closed reduction and cast application, external fixation alone or with percutaneous $\mathrm{K}$ wire fixation were excluded from the study. All patients had intra-articular involvement with volar and dorsal metaphyseal comminution and were classified in Group $\mathrm{C}$ according to the AO/OTA classification. (Fig 1)

\section{Surgical Technique}

Majority of patients were administered an axillary block but six had general anaesthesia and all patient were done under tourniquet control.

An external fixator was initially applied using the technique described. An open technique was used for placement of the fixator pins. Pins were placed in the neck and base of the second metacarpal ${ }^{3}$. The most distal pin in the radius was placed at least $2 \mathrm{~cm}$ from the fracture site, and the proximal pin was placed at least $2 \mathrm{~cm}$ proximal to the distal pin. In all patients an AO external fixator with 3.5/2.5-mm Schanz pins was used with a double-stacked frame was constructed for stability. Longitudinal traction was along the forearm to help reduce the fracture and correct radial length and displacement through ligamentotaxis. The fixator was locked into place and checked under fluoroscopy in posteroanterior, lateral and oblique projections. In all patients the use of external fixation alone was not adequate to reduce articular congruity and to correct and maintain radial length and/or had failure of maintenance of fracture reduction.

Open reduction with the volar approach was made between the flexor carpi radialis tendon and the radial artery ${ }^{10}$. Dissection is extended down to the pronator quadratus, and the muscle is freed from the volar aspect of the distal end of the radius, exposing the fracture ${ }^{7}$. The volar capsule is not dissected off the volar aspect of the radius in order to maintain the strong ligamentous support. The soft tissue release of the volar articular fragments should be limited to the extent necessary to allow visualisation and to ensure anatomic reduction of the articular surface ${ }^{11}$. To correct radial shortening and mal-alignment, the articular surface should be elevated as a unit to the level of the scaphoid and lunate and then reduced ${ }^{7}$. After the articular fragments had been anatomically reduced, all fractures required stabilisation with volar plate with or without additional $\mathrm{K}$ wire fixation depending on fragment size. Three fractures were stabilised with additional $1.8 \mathrm{~mm}$ Kirschner wires (Fig 2). Defects in four fractures were filled with bone graft to provide stability and to prevent collapse $e^{6,12}$. Indications for bone-grafting were fracture in which defects in the metaphysis alone or in the metaphysis and diaphysis were noted after reduction of the articular surface $^{13}$. Corticocancellous iliac crest bone graft was used for all fractures with larger defects. The graft was placed in the defect with the cortex positioned so that it offered optimal resistance to displacement or collapse of the articular fragments. The degree of comminution of the distal fragment decided to keep the plate in the buttress mode or insert screws.

\section{Postoperative Protocol}

All patients were instructed to begin active and passive range of motion exercises of the hand, forearm, elbow and shoulder on the day after the surgery. The sutures were removed on the fourteenth day and the pin tracts were cleaned four/five times every day with antiseptics. The external fixator remained until bonehealing was demonstrated on plain radiographs; it was removed at a mean of seven weeks (range, six to eight weeks) postoperatively. Bone-healing was determined radiographically by the appearance of bridging trabeculae across the fracture site and clinically by the fracture site being nontender to palpation. Additional Kirschner wires were removed in the three patients when the fixator was removed (Fig 3).

\section{Patient Evaluation}

At the time of follow-up the patient were asked about pain, return to previous activity and overall satisfaction. A clinical rating as devised by Cooney ${ }^{14}$ (modified from 
Green an O'Brien) (Table 1) was given to each patient as follows: pain, functional status, range of motion, and grip strength measured as percentage of normal (range, 0-25 points) Points were accumulated for the four categories and a final rating were obtained as follows: excellent, $90-100$ points, good, $80-90$ points; fair, $65-80$ points, and poor, less than 65 points.

\section{Results}

There were 22 patients treated with external fixation and open reduction and plate fixation in this study. Fifteen male and 7 female patients were followed up for a period of 14 to 46 months (av.32 months). The dominant extremity was involved in 16 patients. All patients were in Group $\mathrm{C}$ according to the AO/OTA classification but majority had $\mathrm{C} 2$ type of fractures where the comminution did not extend to the diaphysis.
According to the clinical scoring chart of Green and O'Brien as modified by Cooney, the results were: excellent in $11(50 \%)$, good in $7(31 \%)$, fair in $2(9 \%)$ and poor in $2(9 \%)$ patients. (Figures 4,5$)$ There was no correlation between male and female patients and the functional outcome. There were very few complications reported. One patient had superficial pin tract infection which healed well with a short course of oral antibiotics. Another patient, a 58 year old lady, had complex regional pain syndrome which eventually got better with oral medication and physiotherapy. Two patients had loss of fracture reduction and shifting of plate. These were type $\mathrm{C} 3$ fractures with gross comminution and the volar plate had to be kept in a buttress mode. One patient had tendon irritation and is awaiting plate removal since the fracture had united. She refused further surgery to correct her malunion.

Table 1: Clinical Scoring Chart: Cooney WP (modified from Green and O'Brien)

\begin{tabular}{|c|c|c|}
\hline \multicolumn{3}{|l|}{ Pain (25 points) } \\
\hline & 25 & no pain \\
\hline & 20 & mild occasional \\
\hline & 15 & moderate tolerable \\
\hline & 0 & severe to intolerable \\
\hline \multicolumn{3}{|l|}{ Functional status (25 points) } \\
\hline & 25 & return to regular employment \\
\hline & 20 & restricted employment \\
\hline & 15 & able to work, unemployment \\
\hline & 0 & unable to work because of pain \\
\hline \multirow[t]{5}{*}{ Range of motion (25 points) Percentage of normal } & 25 & $100 \%$ \\
\hline & 15 & $75-100 \%$ \\
\hline & 10 & $50-75 \%$ \\
\hline & 5 & $25-50 \%$ \\
\hline & 0 & $0-25 \%$ \\
\hline \multicolumn{3}{|l|}{ DF-PF arcs if only injured hand reported } \\
\hline & 25 & $120^{0}$ or more \\
\hline & 15 & $90^{\circ}-120^{0}$ \\
\hline & 10 & $60^{0}-90^{0}$ \\
\hline & 5 & $30^{0}-60^{0}$ \\
\hline & 0 & $30^{\circ}$ or less \\
\hline \multicolumn{3}{|l|}{ Grip Strength (25 points) Percentage if normal } \\
\hline & 25 & $100 \%$ \\
\hline & 15 & $75-100 \%$ \\
\hline & 10 & $50-75 \%$ \\
\hline & 5 & $25-50 \%$ \\
\hline & 0 & $0-25 \%$ \\
\hline
\end{tabular}



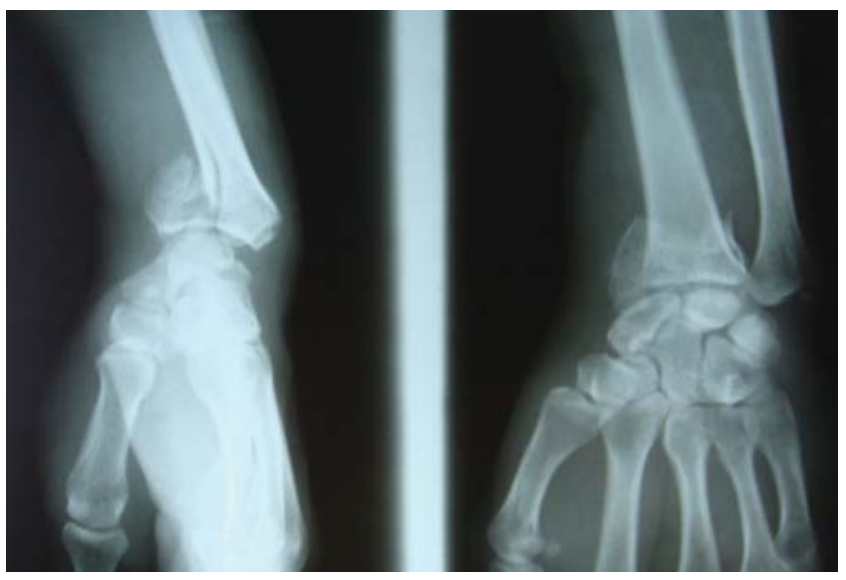

Fig 1: Comminuted distal radius fracture AO/OTA Group C2

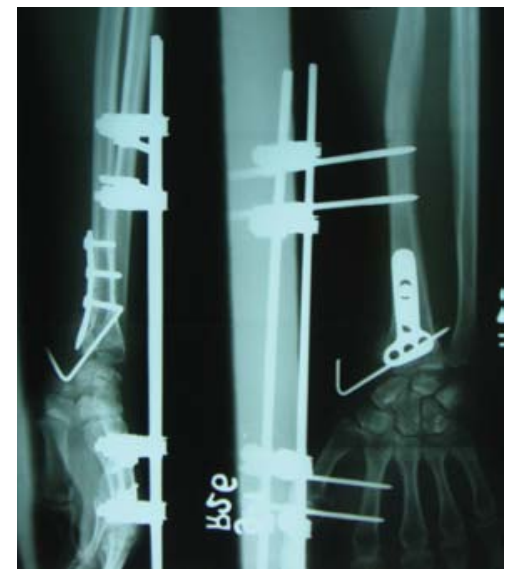

Fig 2: Post-operative radiograph treated with external fixation and volar plate with additional $\mathrm{K}$ wire fixation

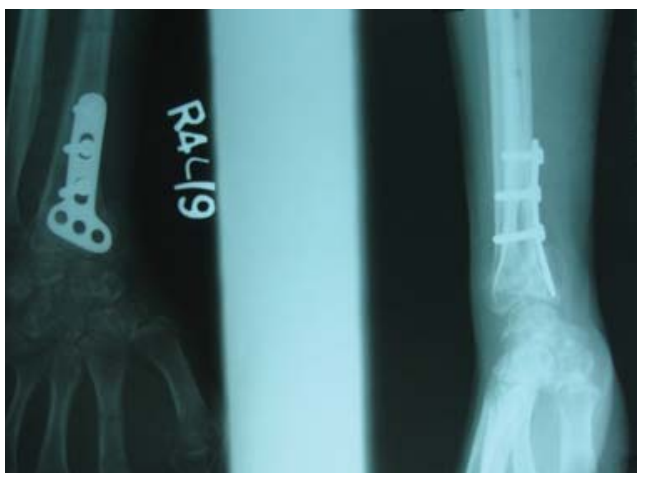

Fig 3: Post-operative radiograph after removal of external fixation and $\mathrm{K}$ wire
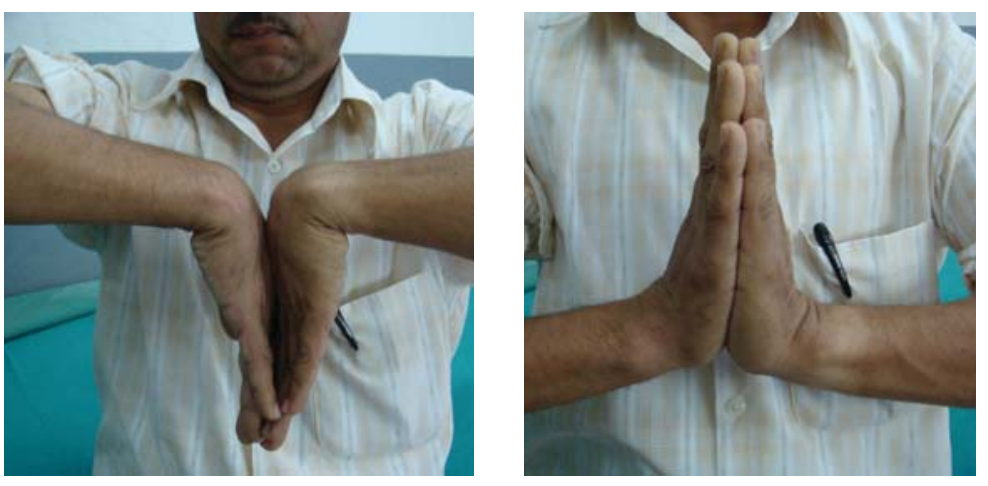

Fig 4: Clinical picture of patients after 24 months followup with excellent results

\section{Discussion}

In optimal management of comminuted distal radial fractures, the magnitude of articular and periarticular soft-tissue disruption must be assessed accurately. Without precise reduction of the displaced joint surfaces, the unstable articular fracture may lead to significant impairment. Our results also indicate that the functional outcome is directly related to the quality of anatomical restoration of the articular surface and extra-articular alignment in comminuted intraarticular fractures of the distal radius as in other studies ${ }^{11,12}$. Failure to achieve proper reduction and length of distal radius relative to ulna can result in complications of pain and instability which may require corrective osteotomy ${ }^{15}$. An articular incongruity of more than $2 \mathrm{~mm}$ may lead to a higher incidence of post-traumatic arthritis and poorer results ${ }^{1}$.

In the management of unstable distal radius fractures, external fixation alone or with percutaneous fixation has produced good or excellent outcomes ${ }^{4,16}$. However, there are still some articular fractures in which articular restoration cannot be corrected and the length maintained through ligamentotaxis alone ${ }^{17}$.

We agree with Rogachefsky et al. ${ }^{18}$ that the augmentation of fracture fixation by external fixator at full distraction before the surgical approach aided in fracture reduction and improved radial length and alignment. The fixator neutralised the compressive forces across the joint, preventing collapse of the fracture during the initial two to four weeks of healing, when bone resorption and early new-bone formation are maximal ${ }^{19}$.

In our study, the external fixator was removed at an average of seven weeks (six to eight) after the operation, earlier than other studies ${ }^{20}$ but in accordance with some $^{18}$. We removed the external fixator early as we had additional fixation in the volar plate and by six to eight weeks the fracture would be relatively stable. The good range of motion achieved in the study could be due 
to this earlier removal as we could mobilise the wrist earlier hence avoiding the complications associated with prolonged immobilisation.

Combined internal and external fixations of comminuted distal radius fractures have reported very high satisfaction results. These studies have used various methods of internal and external fixation. Our study comprises of all patients treated with similar external fixation device used in the same bridging mode and using the conventional volar plate for distal radius supplemented by K-wire in some. Our results are at par with these studies and show that according to Green and O'Brien scoring system $50 \%$ had excellent results and $17 \%$ good in this study. The results of Rogachefsky et al and ours as well are comparable with those in many series that have included less severe fractures.

The poor outcome in the two patients was the ones who had severe comminution and had shifting of the plate. We now believe that if bone grafting had been done then we could have avoided this complication. Hence, we believe that if there is bone loss we should not hesitate to use bone graft or its substitute to support metaphyseal comminution.

Limitations of our study include its lack of controls, its retrospective nature and relatively small number of cases.

\section{Conclusion}

We believe that comminuted intra-articular fractures of distal radius should be treated by open reduction and combined internal and external fixation to achieve a high rate of patient satisfaction and satisfactory functional outcome.

\section{References}

1. Knirk JL, Jupiter JB. Intra-articular fractures of the distal end of the radius in young adults. $\mathrm{J}$ Bone Joint Surg Am.1986; 68:647-59.

2. Nakata RY, Chand Y, Matiko JD, Frykman GK, Wood VE. External fixators for wrist fractures: a biomechanical and clinical study. J Hand Surg [Am].1985;10: 845-51.

3. Jenkins NH, Jones DG, Johnson SR, MintowtCzyz WJ. External fixation of Colles' fractures. An anatomical study. J Bone Joint Surg Br. 1987;69:207-11.

4. Howard PW, Steward HD, Hind RE, Burke FD. External fixation or plaster for severely displaced comminuted Colles' fractures? J Bone Joint Surg Br. 1989; 71 (1): 68-73.

5. Melone CP Jr. Open treatment for displaced articular fractures of the distal radius. Clin Orthop. 1986; 202:103-11.
6. Missakian ML, Cooney WP, Amadio PC, Glidewell HL. Open reduction and internal fixation for distal radius fractures. J Hand Surg [Am]. 1992;17: 745-55.

7. Ruch DS, Papadonikolakis A. J Hand Surg [Am]. 2006;31:9-16.

8. Nana AD, Joshi A, Lichtman DM. Plating of the Distal Radius. J Am Acad Orthop Surg. 13, 159171.

9. Bass RL, Blair WF, Hubbard PP. Results of combined internal and external fixation for the treatment of severe AO-C3 fractures of the distal radius. J Hand Surg [Am]. 1995; 20:373-381.

10. Trumble TE, Schmitt SR, Vedder NB. Factors affecting functional outcome of displaced intraarticular distal radius fractures. J Hand Surg [Am]. 1994;19:325-40.

11. Jupiter JB, Lipton H. The operative treatment of intraarticular fractures of the distal radius. Clin Orthop. 1993;292:48-61.

12. Hove LM, Nilsen PT, Ove F et al. Acta Orthop Scand. 1997, 68 (1) 59-63.

13. Bradway JK, Amadio PC, Cooney WP. Open reduction and internal fixation of displaced, comminuted intra-articular fractures of the distal end of the radius. J Bone Joint Surg Am. 1989;71:839-47.

14. Cooney WP, Bussey R, Dobyns JH, Linscheid RL. Difficult wrist fractures. Perilunate fracturedislocations of the wrist. Clin Orthop. 1987; 214:136-47.

15. Jupiter JB, Masem M. Reconstruction of posttraumatic deformity of the distal radius and ulna. Hand Clin. 1998; 4(3):377-90.

16. Hertel R, Jakob RP. Static external fixation of the wrist Hand Clin. 1993; 9 (4):567-75

17. McAuliffe JA. Combined internal and external fixation of distal radius fractures. Hand Clin. 2005; 21 (3):395-406.

18. Rogachefsky RA, Lipson SR, Applegate B et al. Treatment of severely comminuted intraarticular fractures of the distal end of the radius by open reduction and combined internal and external fixation. . J Bone Joint Surg Am. 2001; 83(4):1241-50.

19. Edwards GS Jr. Intra-articular fractures of the distal part of the radius treated with the small AO external fixator. J Bone Joint Surg Am. 1991;73:1241-50.

20. Yen ST, Hwang CY, Hwang MH. A semiinvasive method for articular Colles' fractures. Clin Orthop 1991; 263: 154-64. 